J. YONG

KODAI MATH. J.

14 (1991), 239-253

\title{
TIME OPTIMAL CONTROLS FOR SEMILINEAR DISTRIBUTED PARAMETER SYSTEMS-EXISTENCE THEORY AND NECESSARY CONDITIONS*
}

\author{
BY JIONGMIN YONG
}

\begin{abstract}
For a semilinear controlled evolution system with state dependent control domain, we study the existence of admissible trajectories, the properties of attainable set as well as the existence of time optimal controls. For the case the control domain is independent of the state, a Pontryagin's type maximum principle is proved.
\end{abstract}

\section{§1. Introduction.}

In this paper, we consider the following controlled evolution system

$$
\dot{x}(t)=A x(t)+f(t, x(t), u(t)),
$$

where $A: \mathscr{D}(A) \subset X \rightarrow X$ generates a $C_{0}$-semigroup $e^{A t}$ on the underline space $X$, $u(t)$ is the control function valued in some metric space $U, x(t)$ is the state of the system valued in $X$ and $f:[0, \infty) \times X \times U \rightarrow X$ is a given map. Let us denote

$$
\mathcal{U}[s, r]=\{u(\cdot):[s, r] \rightarrow U \mid u(\cdot) \text { is measusable }\},
$$

for any $0 \leqq s<r<\infty$. For any $y \in X, 0 \leqq s<r<\infty$ and $u(\cdot) \in U[s, r]$, a function $x(\cdot) \in C([s, r] ; X)$ is called a (mild) solution of (1.1) corresponding to $(y, u(\cdot))$, if it satisfies the following integral equation

$$
x(t)=e^{A(t-s)} y+\int_{s}^{t} e^{A(t-s)} f(\tau, x(\tau), u(\tau)) d \tau, \quad t \in[s, r] .
$$

Now, we are also given (multivalued) maps $\Gamma:[0, \infty) \times X \rightarrow 2^{U}$ and $Q:[0, \infty)$ $\rightarrow 2^{X}$. They will be the (state dependent) control domain and the target set.

Definition 1.1. Let $0 \leqq s<r<\infty$ and $y \in X$. We say $(x(\cdot), u(\cdot)) \in$

* This work was partially supported by the NSF of China under Grant 0188416 and the Chinese State Education Commission Science Foundation under Grant 9024617.

Received May 2, 1990; revised December 3, 1990. 
$C([s, r] ; X) \times \mathscr{U}[s, r]$ is an admissible pair (corresponding to $y \in X)$ on $[s, r]$, if $(1.2)$ is satisfied and

$$
u(t) \in \Gamma(t, x(t)), \quad \text { a.e. } t \in[s, r] .
$$

In this case, we refer $x(\cdot)$ and $u(\cdot)$ admissible trajectory and control, respectively.

We denote

$$
\begin{gathered}
\mathcal{A}([s, r] ; y)=\{(x(\cdot), u(\cdot)) \in C([s, r] ; X) \times \mathcal{U}[s, r] \mid(x(\cdot), u(\cdot) \\
\text { is an admissible pair corresponding to } y \in X\}, \\
\mathscr{X}([s, r] ; y)=\{x(\cdot) \in C([s, r] ; X) \mid(x(\cdot), u(\cdot)) \in \mathcal{A}([s, r] ; y), \\
\text { for some } u(\cdot) \in \mathcal{U}[s, r]\}, \\
\mathscr{R}(r ; s, y)=\{x(r) \mid x(\cdot) \in \mathscr{X}([s, r] ; y)\} .
\end{gathered}
$$

Next, we let

$$
\begin{aligned}
& \mathscr{B}(s ; y)=\left\{(x(\cdot), u(\cdot)) \in \bigcup_{r>s} \mathcal{A}([s, r] ; y) \mid x(\tilde{t}) \in Q(\tilde{t}), \text { for some } \tilde{t} \geqq s\right\}, \\
& \mathscr{I}(x(\cdot))=\{\tilde{t} \in[0, \infty) \mid x(\tilde{t}) \in Q(\tilde{t})\}, \quad \forall x(\cdot) \in \bigcup_{0 \leqq s<r<\infty} C([s, r] ; X) .
\end{aligned}
$$

Then, our time optimal control problem can be stated as follows:

Problem T. Let $\mathscr{B}(s ; y) \neq \varnothing$. Find $\left(x^{*}(\cdot), u^{*}(\cdot)\right) \in \mathscr{B}(s ; y)$ and $t^{*} \in \mathscr{T}\left(x^{*}(\cdot)\right)$, such that

$$
t^{*}=\min _{(x(\cdot), u(\cdot)) \in \mathscr{B}(s, y)} \inf \mathscr{I}(x(\cdot)) .
$$

If $x^{*}(\cdot), u^{*}(\cdot), t^{*}$ exist solving Problem $\mathrm{T}$, we refer $x^{*}(\cdot), u^{*}(\cdot),\left(x^{*}(\cdot), u^{*}(\cdot)\right)$ a time optimal trajectory, control, pair, respectively and $t^{*}$ the minimum hitting time.

Time optimal control problems were studied by many authors. We refer the readers to $[4,17,18]$ for finite dimensional (linear and nonlinear) cases, to $[1,3,10,13]$ for infinite dimensional linear case and to [19] for infinite dimensional semilinear case. We should note that [19] discussed the necessary conditions for a semilinear distributed parameter system with target sets having nonempty interior. Thus, the results of this paper differ those of [19] in the following two aspects. First, we discuss the existence of the admissible trajectories as well as optimal controls for the case of state-dependent control domain. Second, we prove a Pontryagin's type maximum principle for the case of stateindependent control domain and the target sets not necessarily having nonempty interior. Also, the method used in proving the necessary conditions is adopted from [15] and is different from that used in [19]. 


\section{§2. Preliminaries.}

We refer the readers to the Appendix for results about multivalued mappings. Now, we list basic assumptions for our control problem: on $X$.

(H1) $X$ is a reflexive Banach space, $U$ is a Polish space ([5]).

(H2) The operator $A: \mathscr{D}(A) \subset X \rightarrow X$ generates a compact $C_{0}$-semigroup $e^{A t}$

(H3) $f:[0, \infty) \times X \times U \rightarrow X$ is Borel measurable in $(t, x, u)$, continuous in $(x, u)$ and there exists a constant $L>0$, such that

$$
\left\{\begin{array}{l}
|f(t, x, u)-f(t, \hat{x}, u)| \leqq L|x-\hat{x}|, \quad \forall t \in[0, \infty), x, \hat{x} \in X, u \in U, \\
|f(t, x, u)| \leqq L(1+|x|), \quad \forall t \in[0, \infty), x \in X, u \in U .
\end{array}\right.
$$

(H4) $\Gamma:[0, \infty) \times X \rightarrow 2^{U}$ is pseudo-continuous.

(H5) $Q:[0, \infty) \times X \rightarrow 2^{X}$ is pseudo-continuous.

(H6) For almost all $t \in[0, \infty)$,

$$
\bigcap_{\delta>0} \overline{\mathrm{co}} f\left(t, \Re_{\delta}(x), \Gamma\left(\mathscr{I}_{\delta}(t, x)\right)\right)=f(t, x, \Gamma(t, x)),
$$

where $\Re_{\delta}(x)=\{\hat{x} \in X|| x-\hat{x} \mid<\delta\}$ and similar for $\Re_{\delta}(t, x)$.

Following result gives a sufficient condition for (H6).

Proposition 2.1. Let $\Gamma:[0, \infty) \times X \rightarrow 2^{U}$ be upper semicontinuous and take closed set values and let $f(t, x, u)$ be uniformly continuous in $(x, u)$ and $t \in[0, \infty)$. Then, (H6) holds iff $f(t, x, \Gamma(t, x))$ is closed and convex.

Proof. It suffices to prove the sufficiency. By the uniform continuity of $f(t, x, u)$ in $(x, u)$, for any $\varepsilon>0$, there exists a $\sigma>0$, such that

$$
f\left(t, \Re_{\sigma}(x), \Re_{\sigma}(\Gamma(t, x))\right) \subset \Re_{\varepsilon}(f(t, x, \Gamma(t, x))) .
$$

Then, by the upper semicontinuous of $\Gamma$, there exists a $\delta$ with $0<\delta \leqq \sigma$, such that

$$
\Gamma\left(\Re_{\delta}(t, x)\right) \subset \Re_{\sigma}(\Gamma(t, x))
$$

Then, (2.2) follows.

Remark 2.2. (H6) is a sort of Cesari property $([1,4])$.

Remark 2.3. In [1], the existence theory of optimal controls for the distributed parameter systems was given under the framework of strong solutions of the evolution equations. It was asked if one can do the same thing under the framework of mild solutions. This paper actually gives a positive answer for the time optimal control problems of a class of distributed parameter systems. For various other optimal control problems of distributed parameter sys- 
tems, the above approach will also work. We will carry out the full details as well as some related problems in a forthcoming paper.

\section{§3. Existence of Adimissible Trajectories and Time Optimal Controls.}

In this section, we first establish the existence of adimissible trajectories. Then, we will discuss the existence of time optimal controls.

TheOREm 3.1. Let (H1)-(H4) and (H6) hold. Then, for any $y \in X, 0 \leqq s \leqq r<\infty$,

$$
\mathscr{X}([s, r] ; y) \neq \varnothing \text {. }
$$

Moreover, the set $\mathfrak{X}([s, r] ; y)$ is compact in $C([s, r] ; X)$.

We note that in the case $\Gamma(t, x) \equiv \Gamma(t), \forall(t, x) \in[0, \infty) \times X$, i. e., the control domain is independent of the state $x$, then, to get (3.1), we only need that $\Gamma(\cdot):[0, \infty) \rightarrow 2^{U}$ is measurable, $A$ generates a $C_{0}$-semigroup on $X$ and (2.1) holds. However, in the case $\Gamma(t, x)$ depends on $x$, the problem becomes a little complicated. To prove the above Theorem 3.1, we need the following lemma.

LeMmA 3.2. Let $e^{A t}$ be a compact $C_{0}$-semigroup on $X$. Then, for any $p>1$, the operator

$$
S(g(\cdot))=\int_{s} e^{A(\cdot-r)} g(\tau) d \tau, \quad \forall g(\cdot) \in L^{p}(s, r ; X),
$$

is a compact operator from $L^{p}(s, r ; X)$ to $C([s, r] ; X)$.

Proof. Without loss of generality, we let $[s, r]=[0,1]$. Next, we let $g_{k}(\cdot) \in L^{p}(0,1 ; X)$ with

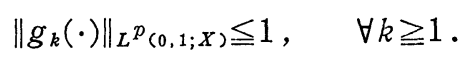

We need to prove that $\left\{S\left(g_{k}(\cdot)\right)\right\}_{k \geqq 1}$ is relatively compact in $C([0,1] ; X)$. To this end, we first claim that for each $t \in[0,1]$, the set $\left\{S\left(g_{k}(\cdot)\right)(t)\right\}_{k \geq 1}$ is relatively compact in $X$. In fact, the case $t=0$ is trivial. Let $t \in(0,1]$. Then, for any $\varepsilon>0$, we can find a $\delta$ with $0<\delta \leqq t$, such that

$$
\left|\int_{t-\delta}^{t} e^{A(t-r)} g_{k}(\tau) d \tau\right|<\frac{\varepsilon}{2}, \quad \forall k \geqq 1
$$

Next, it is clear that if we put

$$
y_{k} \equiv \int_{0}^{t-\delta} e^{A(t-\delta-r)} g_{k}(\tau) d \tau, \quad \forall k \geqq 1,
$$

then, the set $\left\{y_{k}\right\}_{k \geq 1}$ is bounded in $X$. Thus by the compactness of $e^{A \delta}$, we can find a finite set $\left\{z_{\imath}, 1 \leqq i \leqq m\right\}$ in $X$, such that 


$$
\left\{e^{A \delta} y_{k}\right\}_{k \geqq 1} \subset \bigcup_{\imath=1}^{m} \eta_{\varepsilon / 2}\left(z_{\imath}\right)
$$

Then, we see that

$$
\left\{S\left(g_{k}(\cdot)\right)(t)\right\}_{k \geqq 1} \subset \bigcup_{\imath=1}^{m} \Re_{\varepsilon}\left(z_{\imath}\right) .
$$

Hence, for each $t \in[0,1],\left\{S\left(g_{k}(\cdot)\right)(t)\right\}_{k \geqq 1}$ is relatively compact in $X$. Next, we show that $\left\{S\left(g_{k}(\cdot)\right)\right\}_{k \geq 1}$ is equicontinuous on $[0,1]$. In fact, for $t^{\prime}>t>0$ and $0<\delta \leqq t$, we have

$$
\begin{aligned}
S\left(g_{k}(\cdot)\right)\left(t^{\prime}\right)-S\left(g_{k}(\cdot)\right)(t)= & \int_{t}^{t^{\prime}} e^{A\left(t^{\prime}-r\right)} g_{k}(\tau) d \tau \\
& +\int_{0}^{t-\delta}\left(e^{A\left(t^{\prime}-r\right)}-e^{A(t-r)}\right) g_{k}(\tau) d \tau \\
& +\int_{t-\delta}^{t}\left(e^{A\left(t^{\prime}-r\right)}-e^{A(t-r)}\right) g_{k}(\tau) d \tau \\
\equiv & I_{1}+I_{2}+I_{3} .
\end{aligned}
$$

It is easy to see that for some constant $C$,

$$
\begin{aligned}
& \left|I_{1}\right| \leqq C\left|t^{\prime}-t\right|^{p /(p-1)}, \\
& \left|I_{2}\right| \leqq C \int_{\delta}^{t}\left\|e^{A\left(t^{\prime}-t+r\right)}-e^{A r}\right\| d \tau, \\
& \left|I_{3}\right| \leqq C \delta .
\end{aligned}
$$

Thus, by the fact that $e^{A t}$ in continuous in the operator topology in $(0, \infty)([16])$, we obtain the equi-continuity of the set $\left\{S\left(g_{k}(\cdot)\right)\right\}$ on $[0,1]$. Then by ArzelaAscoli Theorem ([20]), we have our conclusion.

Proof of Theorem 3.1. Again, we only prove the case $[s, r]=[0,1]$. For any $k \geqq 1$, let

$$
t_{j}=\frac{j}{k}, \quad 0 \leqq j \leqq k
$$

We set

$$
u_{k}(t)=\sum_{j=1}^{k-1} u^{\jmath} \chi_{\left[t_{j}, t_{j+1}\right)}(t), \quad t \in[0,1] .
$$

Here, $u^{\prime}$ 's are constructed as follows: First, we take

$$
u^{0} \in \Gamma(0, y) .
$$

By (2.1), we know that there exists a unique $x_{k}(\cdot)$ satisfies 


$$
x_{k}(t)=e^{A t} y+\int_{0}^{t} e^{A(t-r)} f\left(r, x_{k}(\tau), u^{0}\right) d \tau, \quad \forall t \in\left[t_{0}, t_{1}\right] .
$$

Then, we take

$$
u^{1} \in \Gamma\left(t_{1}, x_{k}\left(t_{1}\right)\right) .
$$

We can continue the above procedure to obtain $x_{k}(\cdot)$ on $\left[t_{1}, t_{2}\right]$, etc. By induction, we end up with the following:

$$
\left\{\begin{array}{l}
x_{k}(t)=e_{A}^{t} y+\int_{0}^{t} e^{A(t-r)} f\left(\tau, x_{k}(\tau), u_{k}(\tau)\right) d \tau, \quad \forall t \in[0,1], \\
u_{k}(t) \in \Gamma\left(t_{j}, x_{k}\left(t_{j}\right)\right), \quad t \in\left[t_{1}, t_{j+1}\right), \quad 0 \leqq j \leqq k-1 .
\end{array}\right.
$$

By Gronwall's inequality and (2.1), we see that

$$
\begin{gathered}
\left|x_{k}(t)\right| \leqq C, \quad \forall t \in[0,1], \quad k \geqq 1 . \\
\left|f\left(t, x_{k}(t), u_{k}(t)\right)\right| \leqq C, \quad \text { a.e. } t \in[0,1], \quad k \geqq 1 .
\end{gathered}
$$

By Lemma 3.2, we know that $\left\{x_{k}(\cdot)\right\}_{k \geqq 1}$ is relatively compact in $C([0,1] ; X)$. Then we may assume

$$
x_{k}(\cdot) \stackrel{s}{\longrightarrow} \bar{x}(\cdot), \quad \text { in } C([0,1] ; X),
$$

for some $\bar{x}(\cdot) \in C([0,1] ; X)$. Also, we may let

$$
f\left(\cdot, x_{k}(\cdot), u_{k}(\cdot)\right) \stackrel{w}{\longrightarrow} \bar{f}(\cdot), \quad \text { in } L^{p}(0,1 ; X),
$$

for some $\bar{f}(\cdot) \in L^{p}(0,1 ; X)$. By the compactness of the operator $S$, we have

$$
\bar{x}(t)=e^{A t} y+\int_{0}^{t} e^{A(t-r)} \bar{f}(r) d r, \quad t \in[0,1] .
$$

By (3.10), for any $\delta>0$, there exists a $k_{0}$, such that

$$
x_{k}(t) \in \Re_{\delta}(\bar{x}(t)), \quad \forall t \in[0,1], \quad k \geqq k_{0} .
$$

On the other hand, by the definition of $u_{k}(\cdot)$, one may assume

$$
u_{k}(t) \in \Gamma\left(t_{\jmath}, x_{k}\left(t_{j}\right)\right) \subset \Gamma\left(\Re_{\delta}(t, \bar{x}(t))\right), \quad \forall t \in\left[t_{\jmath}, t_{j+1}\right), \quad 0 \leqq j \leqq k-1 .
$$

Next, by (3.11) and Mazur Theorem, there exist $\alpha_{\imath \jmath} \geqq 0(j \geqq 1$ and finitely many $i$ for each $j$ ) with $\sum_{\imath \geqq 1} \alpha_{\imath \jmath}=1$, such that for some $p<\infty$,

$$
\phi_{j}(\cdot) \equiv \sum_{i \geqq 1} \alpha_{\imath j} f\left(\cdot, x_{i}(\cdot), u_{i}(\cdot)\right) \stackrel{s}{\longrightarrow} \bar{f}(\cdot), \quad \text { in } L^{p}(0,1 ; X) .
$$

Then, we may assume

$$
\phi_{j}(t) \stackrel{s}{\longrightarrow} \bar{f}(t), \quad \text { in } X, \text { a. e. } t \in[0,1] .
$$


On the other hand, by (3.13) and (3.14), we see that for $\jmath$ large,

$$
\phi_{j}(t) \in \operatorname{co} f\left(t, \Re_{\delta}(\bar{x}(t)), \Gamma\left(\Re_{\delta}(t, \bar{x}(t))\right)\right), \quad \text { a.e. } t \in[0,1] .
$$

Thus, for any $\delta>0$, we have

$$
\bar{f}(t) \in \overline{\mathrm{co}} f\left(t, \Re_{\delta}(\bar{x}(t)), \Gamma\left(\Re_{\delta}(t, \bar{x}(t))\right)\right), \quad \text { a. e. } t \in[0,1] .
$$

By (H6), we get

$$
\bar{f}(t) \in f(t, \bar{x}(t), \Gamma(t, \bar{x}(t))), \quad \text { a.e. } t \in[0,1] .
$$

By Theorems A.3 and A.4 of the Appendix, we know that there exists a $\bar{u}(\cdot)$ $\in \mathcal{V}[0,1]$, such that

$$
\left\{\begin{array}{l}
\bar{u}(t) \in \Gamma(t, \bar{x}(t)), \quad \text { a.e. } t \in[0,1], \\
\bar{f}(t)=f(t, \bar{x}(t), \bar{u}(t)), \quad \text { a.e. } t \in[0,1] .
\end{array}\right.
$$

Combining (3.20) and (3.12), we see that

$$
(\bar{x}(\cdot), \bar{u}(\cdot)) \in \mathcal{A}([0,1] ; y) .
$$

Thus, (3.1) follows. Finally, let $\left\{x^{k}(\cdot)\right\}_{k \geqq 1} \subset \mathscr{X}([0,1] ; y)$ with

$$
\left\|x^{k}(\cdot)\right\|_{C([0,1] ; X)} \leqq C, \quad \forall k \geqq 1 .
$$

Then, as in the above proof, we see that $\left\{x^{k}(\cdot)\right\}_{k \geqq 1}$ is relatively compact in $C([0,1] ; X)$. Moreover, if for some subsequence (still denoted by itself), one has

$$
x^{k}(\cdot) \stackrel{s}{\longrightarrow} \hat{x}(\cdot), \quad \text { in } C([0,1] ; X) .
$$

Again, by $(\mathrm{H} 6)$, we have $\hat{x}(\cdot) \in \mathscr{X}([0,1] ; y)$. Thus, $\mathscr{X}([0,1] ; y)$ is compact in $C([0,1] ; X)$.

CoROllary 3.3. Let (H1)-(H4) and (H6) hold. Then for any $y \in X$ and $0 \leqq s \leqq r<\infty$, the set $\mathcal{R}(r, s, y)$ is nonempty and compact in $X$.

Proposition 3.4. Let (H1)-(H4) and (H6) hold. Then, for any $y \in X$ and $s \in[0, \infty)$, the map $R(\cdot ; s, y):[s, \infty) \rightarrow 2^{X}$ is continuous (with respect to the Hausdorff metric $\rho_{H}$ ).

Proof. By Theorem 3.1, we know that for the given $y \in X, s \in[0, \infty)$ and any $r \in[s, \infty)$, we can find a continuous, nondecreasing function $\omega:[0, \infty) \rightarrow$ $[0, \infty)$ with $\omega(0)=0$, such that

$$
\left|x(t)-x\left(t^{\prime}\right)\right| \leqq \omega\left(\left|t-t^{\prime}\right|\right), \quad t, t^{\prime} \in[s, r], \quad x(\cdot) \in \mathscr{X}([s, r] ; y) .
$$

Then, it is easy to see that 


$$
\rho_{H}\left(\mathscr{R}(t ; s, y), \mathscr{R}\left(t^{\prime} ; s, y\right)\right) \leqq \omega\left(\left|t-t^{\prime}\right|\right), \quad \forall t, t^{\prime} \in[s, r] .
$$

This proves the continuity of the map $\mathscr{R}(\cdot ; s, y)$.

Theorem 3.5. Let (H1)-(H6) hold. Then, Problem $T$ admits at least one solution.

Proof. Let $x_{0} \in X,\left(x_{k}(\cdot), u_{k}(\cdot)\right) \in \mathscr{B}\left(0 ; x_{0}\right), \tilde{t}_{k} \in \mathscr{I}\left(x_{k}(\cdot)\right)$ and

$$
\lim _{k \rightarrow \infty} \tilde{t}_{k}=t^{*} \equiv \inf _{(x(\cdot), u(\cdot)) \in \mathscr{B}\left(0 ; x_{0}\right)} \inf \mathscr{I}(x(\cdot)) .
$$

Thus, by the definition of $\mathscr{I}\left(x_{k}(\cdot)\right)$, we have

$$
x_{k}\left(\tilde{t}_{k}\right) \in Q\left(\tilde{t}_{k}\right) \cap \mathscr{R}\left(\tilde{t}_{k} ; 0, x_{0}\right), \quad \forall k \geqq 1 .
$$

On the other hand, by Theorem 3.1, we may assume that all $x_{k}(\cdot)$ 's are defined on $\left[0, \tilde{t}_{1}\right]$ and we may also let

$$
x_{k}(\cdot) \stackrel{s}{\longrightarrow} \bar{x}(\cdot), \quad \text { in } C\left(\left[0, \tilde{t}_{1}\right] ; X\right),
$$

for some $\bar{x}(\cdot) \in \mathscr{X}\left(\left[0, \tilde{t}_{1}\right] ; x_{0}\right)$. As a consequence, we have

$$
x_{k}\left(\tilde{t}_{k}\right) \stackrel{s}{\longrightarrow} \bar{x}\left(t^{*}\right), \quad \text { in } X .
$$

By Proposition 3.4 and (3.24), we have

$$
\bar{x}\left(t^{*}\right) \in R\left(t^{*} ; 0, x_{0}\right) .
$$

It follows from (3.23) that for any $\delta>0$, provided $k$ large enough, one has

$$
x_{k}\left(\tilde{t}_{k}\right) \in Q\left(\tilde{t}_{k}\right) \subset Q\left(\Re_{\delta}\left(t^{*}\right)\right) .
$$

Thus, by the pseudo-continuity of $Q(\cdot)$, we have

$$
\bar{x}\left(t^{*}\right) \in \bigcap_{\delta>0} \overline{Q\left(\Re_{\delta}\left(t^{*}\right)\right)}=Q\left(t^{*}\right) .
$$

which gives,

$$
\bar{x}\left(t^{*}\right) \in \mathscr{R}\left(t^{*} ; 0, x_{0}\right) \cap Q\left(t^{*}\right),
$$

and our conclusion follows.

\section{$\S 4$. Necessary Conditions for Time Optimal Controls}

In this section, we prove a Pontryagin type maximum principle for our time optimal control problem. To this end, let us first make the following assumptions :

(M1) $X$ is a Banach space with strictly convex dual $X^{*}, U$ is a metric 
space.

(M2) $A: \mathscr{D}(A) \subset X \rightarrow X$ generates a $C_{0}$-semigroup $e^{A t}$ on $X$.

(M3) $f:[0, \infty) \times X \times U \rightarrow X$ is measurable in $t$, continuously Frechet differentiable in $x$ and continuous in $u$. Moreover, $f_{x}(t, x, u)$ is bounded.

(M4) $\Gamma:[0, \infty) \rightarrow 2^{U}$ is measurable (see Appendix).

(M5) $Q:[0, \infty) \rightarrow 2^{X}$ is continuous (with respect to $\rho_{H}$ ) taking closed convex set values.

We define

$$
\mho_{a d}=\left\{u(\cdot):[0, \infty) \rightarrow U \mid u(\cdot) \text { is a measurable selection of } I^{\prime}(\cdot)\right\} .
$$

Sometimes, by $\mathcal{V}_{a d}[a, b]$ we mean the elements $\mathcal{V}_{a d}$ restricted to the interval $[a, b]$. It is clear that under (M2)-(M3), for any $x_{0} \in X$ and $u(\cdot) \in \mathcal{U}_{a d}$, there exists a unique solution of

$$
x(t)=e^{A t} x_{0}+\int_{0}^{t} e^{A(t-r)} f(\tau, x(\tau), u(\tau)) d \tau, \quad t \in[0, \infty) .
$$

Thus, $\left(x_{0}, u(\cdot)\right) \mapsto x(\cdot)$ is a well-defined map from $X \times \mathcal{V}_{a d}$ to $C([0, \infty) ; X)$. Sometimes we use the notation $x\left(\cdot ; u(\cdot), x_{0}\right)$ or $x(\cdot ; u(\cdot))$ to indicate the corresponding dependence. It should be pointed out that the conditions on the map $f$ can be slightly relaxed. But we prefer not to give the most general one.

We find that due to the different nature of the existence theory and the necessary conditions, the sets $(\mathrm{H} 1)-(\mathrm{H} 6)$ and $(\mathrm{M} 1)-(\mathrm{M} 5)$ are quite different. From [2], we know that if $X$ is reflexive or separable, then one can renorm the space so that the dual $X^{*}$ is stractly convex.

Now, we let $(\bar{x}(\cdot), \bar{u}(\cdot))$ be an optimal pair with $\bar{t}$ being the optimal hitting time. We set

$$
\begin{aligned}
& \hat{\mathcal{R}}(\bar{t})=\{\xi(\bar{t}) \mid \xi(t)= \int_{0}^{t} e^{A(t-r)} f_{x}(r, \bar{x}(r), \bar{u}(r)) \xi(r) d r \\
&+\int_{0}^{t} e^{A(t-r)}[f(r, \bar{x}(r), u(r))-f(r, \bar{x}(r), \bar{u}(r))] d r \\
&\left.\forall t \in[0, t], \quad u(\cdot) \in \mathcal{U}_{a d}\right\} .
\end{aligned}
$$

We usually refer the set $\hat{R}(\cdot)$ the reachable set of the variation system alone the pair $(\bar{x}(\cdot), \bar{u}(\cdot))$. Following notion will be necessary.

Definition 4.1. A subset $K \subset X$ is said to be finite codimensional if for some $x \in \overline{\mathrm{co}} K, \operatorname{span}\{\overline{\mathrm{co}} K-x\}$ is a finite codimensional subspace of $X$ and $\overline{\mathrm{co}} K-x$ has nonempty relative interior part in $\operatorname{span}\{\overline{\mathrm{co}} K-x\}$.

Our main result of this section is the following

Theorem 4.2 (Maximum Principle). Let (M1)-(M5) hold. Let $(\bar{x}(\cdot), \bar{u}(\cdot), \bar{t})$ be optimal. Suppose $\hat{\mathcal{R}}(\bar{t})-Q(\bar{t})$ is of finite codimension. Then, there exists a 
$\phi(\cdot) \neq 0$, such that

$$
\begin{aligned}
& \psi(t)=e^{A *(\bar{t}-t)} \psi(\bar{t})+\int_{t}^{\bar{t}} e^{A *(r-t)} f_{x}(r, \bar{x}(r), \bar{u}(r))^{*} \psi(r) d r, \quad t \in[0, t], \\
& \int_{0}^{\bar{t}}\langle\psi(t), f(t, \bar{x}(t), \bar{u}(t))\rangle d t=\max _{u(\cdot) \in \mathcal{U}_{a d}} \int_{0}^{\bar{t}}\langle\psi(t), f(t, \bar{x}(t), u(t))\rangle d t,
\end{aligned}
$$

and

$$
\langle\psi(\bar{t}), q-\bar{x}(\bar{t})\rangle \geqq 0, \quad \forall q \in Q(\bar{t}) .
$$

Moreover, if $U$ is a Polish space, then, (4.4) becomes

$$
\langle\psi(t), f(t, \bar{x}(t), \bar{u}(t))\rangle=\max _{u \in \Gamma_{(}(t)}\langle\psi(t), f(t, \bar{x}(t), u)\rangle, \quad \text { a.e. } t \in[0, T] .
$$

Proof. Let $T>\bar{t}$ be a fixed constant. We define

$$
d(u(\cdot), \hat{u}(\cdot))=\operatorname{meas}\{t \in[0, T]: u(t) \neq \hat{u}(t)\}, \quad \forall u(\cdot), \hat{u}(\cdot) \in \bigcup_{a d} .
$$

Then, similar to $[11,15]$, we see that $\left(\mathcal{U}_{a d}[0, T], d\right)$ is a complete metric space. Next, we define

$$
\begin{aligned}
F_{\varepsilon}(u(\cdot)) & =d_{Q(\bar{t}-\varepsilon)}(x(\bar{t}-\varepsilon ; u(\cdot))) \\
& \equiv \inf _{y \in Q(\bar{t}-\varepsilon)}\|x(\bar{t}-\varepsilon ; u(\cdot))-y\|, \quad \forall u(\cdot) \in \mathcal{U}_{a d} .
\end{aligned}
$$

It is clear that $F_{\varepsilon}(\cdot)$ is continuous on $\left(\bigcup_{a d}[0, T], d\right)$. Also, by the optimality of $(\bar{x}(\cdot), \bar{u}(\cdot), \bar{t}), F_{\varepsilon}(\cdot)$ is strictly positive on $U_{a d}[0, T]$. Moreover, (note $\bar{x}(\cdot)=$ $x(\cdot, \bar{u}(\cdot)))$

$$
F_{\varepsilon}(\bar{u}(\cdot))=d_{Q(\bar{t}-\varepsilon)}(\bar{x}(\bar{t}-\varepsilon)) \equiv \sigma(\varepsilon) \longrightarrow 0, \quad \varepsilon \rightarrow 0 .
$$

Thus, by Ekeland's variational principle $([9])$, one can find a $u^{\varepsilon}(\cdot) \in \mathcal{U}_{a d}[0, T]$, such that

$$
\left\{\begin{array}{l}
F_{\varepsilon}\left(u^{\varepsilon}(\cdot)\right) \leqq F_{\varepsilon}(\bar{u}(\cdot)), \\
d\left(u^{\varepsilon}(\cdot), \bar{u}(\cdot)\right) \leqq \sqrt{\sigma(\varepsilon)} \\
F_{\varepsilon}(u(\cdot))-F_{\varepsilon}\left(u^{\varepsilon}(\cdot)\right) \geqq-\sqrt{\sigma(\varepsilon)} d\left(u(\cdot), u^{\varepsilon}(\cdot)\right) .
\end{array}\right.
$$

Now, we let $u(\cdot) \in \mathcal{U}_{a d}[0, T]$ be fixed. Then, as in $[14,15]$, for any $\rho \in$ $(0,1]$, there exists a measurable set $E_{\rho} \subset[0, T]$, such that meas $E_{\rho}=\rho T$ and if one defines

$$
u_{\rho}^{\varepsilon}(\cdot)=u^{\varepsilon}(\cdot) \chi_{[0, T] \backslash E_{\rho}}(\cdot)+u(\cdot) \chi_{E_{\rho}}(\cdot),
$$

then, $u_{\rho}^{\varepsilon}(\cdot) \in \mathcal{U}_{a d}[0, T]$ and the trajectory $x_{\sigma}^{\varepsilon}(\cdot)$ of $(4.1)$ corresponding to $\left(x_{0}, u_{\sigma}^{\varepsilon}(\cdot)\right)$ satisfies

$$
x_{\sigma}^{\varepsilon}(t)=x^{\varepsilon}(t)+\rho \xi_{\varepsilon}(t)+o(\rho), \quad \text { uniformly in } t \in[0, T],
$$


where $\xi_{s}(\cdot)$ satisfies the following variation system

$$
\begin{aligned}
\xi_{\varepsilon}(t)=\int_{0}^{t} e^{A(t-r)} f_{x}\left(r, x^{\varepsilon}(r), u^{\varepsilon}(r)\right) \xi_{\varepsilon}(r) d r \\
\quad+\int_{0}^{t} e^{A(t-r)}\left[f\left(r, x^{\varepsilon}(r), u(r)\right)-f\left(r, x^{\varepsilon}(r), u^{\varepsilon}(r)\right)\right] d r, \quad \forall t \in[0, T] .
\end{aligned}
$$

Then, as in [15], under (M1) and by the convexity of $Q(\bar{t}-\varepsilon)$, we know that

$$
\partial d_{Q(\bar{t}-\varepsilon)}\left(x^{\varepsilon}(\bar{t}-\varepsilon)\right) \equiv\left\{\phi^{\varepsilon}\right\},
$$

is a singleton and moreover

$$
\left|\phi^{\varepsilon}\right|_{X *}=1
$$

Then, by (4.10), we obtain

$$
-\sqrt{\sigma(\varepsilon)} \leqq\left\langle\phi^{\varepsilon}, \xi_{\varepsilon}(\bar{t}-\varepsilon)\right\rangle,
$$

and

with

$$
\xi_{\varepsilon}(t) \longrightarrow \xi(t) \quad \text { as } \varepsilon \rightarrow 0, \quad \text { uniformly in } t \in[0, T] \text {, }
$$

$$
\begin{aligned}
\xi(t)= & \int_{0}^{t} e^{A(t-r)} f_{x}(r, \bar{x}(r), \bar{u}(r)) \xi(r) d r \\
& +\int_{0}^{t} e^{A(t-r)}[f(r, \bar{x}(r), u(r))-f(r, \bar{x}(r), \bar{u}(r))] d r, \quad \forall t \in[0, T] .
\end{aligned}
$$

Thus, combining (4.14), we have

$$
\left\langle\psi^{\varepsilon}, \xi(\bar{t})\right\rangle \geqq-\left|\xi(\bar{t})-\xi_{\varepsilon}(\bar{t}-\varepsilon)\right|-\sqrt{\sigma(\varepsilon)} .
$$

On the other hand, by the convexity of $Q(\bar{t}-\varepsilon)$, one has

$$
\left\langle\phi^{\varepsilon}, y-x^{\varepsilon}(\bar{t}-\varepsilon)\right\rangle \leqq d_{Q(\bar{t}-\varepsilon)}(y)-d_{Q(\bar{t}-\varepsilon)}\left(x^{\varepsilon}(\bar{t}-\varepsilon)\right), \quad \forall y \in X .
$$

Thus, for all $y \in X$, we have

$$
\begin{aligned}
\left\langle\psi^{\varepsilon}, y-\bar{x}(\bar{t})\right\rangle & =\left\langle\phi^{\varepsilon}, x^{\varepsilon}(\bar{t}-\varepsilon)-\bar{x}(\bar{t})\right\rangle+\left\langle\psi^{\varepsilon}, y-\xi^{\varepsilon}(\bar{t}-\varepsilon)\right\rangle \\
& \leqq\left|x^{\varepsilon}(\bar{t}-\varepsilon)-\bar{x}(\bar{t})\right|+d_{Q(\bar{t}-\varepsilon)}(y) \\
& \leqq\left|x^{\varepsilon}(\bar{t}-\varepsilon)-\bar{x}(\bar{t})\right|+\rho_{H}(Q(\bar{t}-\varepsilon), Q(\bar{t})) .
\end{aligned}
$$

From (4.17) and (4.18), we obtain

$$
\left\langle\phi^{\varepsilon}, \xi-(y-\bar{x}(\bar{t}))\right\rangle \geqq-\delta_{\varepsilon}, \quad \forall \xi \in \hat{\mathscr{R}}(\bar{t}), y \in Q(\bar{t}), \quad \delta_{\varepsilon} \rightarrow 0 .
$$

Hence, if $\hat{R}(\bar{t})-Q(\bar{t})$ is of finite codimension, then, as in $[11,15]$, we can find a subsequence of $\left\{\psi^{\varepsilon}\right\}$ (still denoted by itself), such that 


$$
\phi^{\varepsilon} \longrightarrow \bar{\phi} \neq 0, \quad \varepsilon \rightarrow 0 .
$$

Then, from the above, one has

$$
\begin{gathered}
\langle\bar{\psi}, \xi(\bar{t})\rangle \geqq 0, \\
\langle\bar{\psi}, y-\bar{x}(\bar{t})\rangle \leqq 0, \quad \forall y \in Q(\bar{t}) .
\end{gathered}
$$

Now, we let

$$
\phi(t)=-e^{A *(\bar{t}-t)} \bar{\psi}+\int_{0}^{\bar{t}} e^{A *(r-t)} f_{x}(r, \bar{x}(r), \bar{u}(r))^{*} \psi(r) d r, \quad t \in[0, t] .
$$

Then, one can easily check that

$$
0 \geqq\langle\psi(\bar{t}), \xi(\bar{t})\rangle=\int_{0}^{\bar{t}}\langle\psi(r), f(r, \bar{x}(r), u(r)-f(r, \bar{x}(r), \bar{u}(r))\rangle d r .
$$

This gives (4.4), and (4.5) follows from (4.22) easily. By (4.20), we see the costate $\psi(\cdot)$ is nonzero. Finally, we prove (4.6) in the case $U$ is a Polish space. To this end, we first note that $(\bar{x}(\cdot), \bar{u}(\cdot))$ is an optimal pair for the problem with $\Gamma(t)$ replaced by $\overline{\Gamma(t)}$. Then, by the above proof, we see that

$$
\int_{0}^{\bar{t}}\langle\psi(r), f(r, \bar{x}(r), u(r))\rangle d r \leqq \int_{0}^{\bar{t}}\langle\psi(t), f(r, \bar{x}(r), \bar{u}(r))\rangle d r,
$$

for all measurable $u(\cdot):[0, \bar{t}] \rightarrow U$ with $u(t) \in \overline{\Gamma(t)}$ a.e. $t \in[0, T]$.

Now, if (4.6) were not true, then, there exists a set $E \subset[0, T]$ with positive Lebesgue measure, such that for some $\delta>0$, we have

$$
\sup _{u \in \bar{\Gamma}(t)}\langle\psi(t), f(t, \bar{x}(t), u)\rangle \geqq\langle\psi(t), f(t, \bar{x}(t), \bar{u}(t))\rangle+2 \delta, \quad \forall t \in E .
$$

Then, we set

$$
\Lambda(t)=\{u \in \overline{\Gamma(t)} \mid\langle\psi(t), f(t, \bar{x}(t), u)\rangle \geqq\langle\psi(t), f(t, \bar{x}(t), \bar{u}(t))\rangle+\delta\}, \quad t \in E .
$$

It is not hard to show that $\Lambda: E \rightarrow 2^{U}$ is measurable and taking closed-set values. Thus, by Theorem A.3, we can find a measurable selection $\bar{v}(\cdot)$ of $\Lambda(\cdot)$. Then, by taking

$$
u(t)=\bar{u}(t) \chi_{[0, T] \backslash E}(t)+\bar{v}(t) \chi_{E}(t), \quad \bar{t} \in[0, T]
$$

in (4.25), we end up with a contradiction. Thus, (4.6) follows.

To close this section we state the following result, the proof of which is easy.

Proposition 4.3. Let $K$ be a subset of finite codimension in some Banach space $X$. Then, for any subset $S$ of $X$, the set $K-S$ is of finite codimension.

From the above Proposition, we see that in Theorem 4.2, the condition that 
$\hat{\mathcal{R}}(t)-Q(\bar{t})$ being of finite codimension holds provided either $\hat{\mathcal{R}}(\bar{t})$ or $Q(\bar{t})$ is of finite codimension.

\section{Appendix. Multivalued Mappings.}

In this appendix, we recall some results about multivalued mappings. First, we recall the following definition.

Definition A.1. Let $T$ and $Z$ be metric spaces, $A: T \rightarrow 2^{Z} \equiv\{$ nonempty subsets of $Z$ \}.

(i) $\Lambda$ is said to be continuous (with respect to the Hausdorff metric $\rho_{H}$ ) at $t \in T$, if

$$
\lim _{\bar{t} \rightarrow t} \rho_{H}(\Lambda(\bar{t}), \Lambda(t))=0 .
$$

(ii) $\Lambda$ is said to be upper semi-continuous at $t \in T$, if for any $\varepsilon>0$, there exists a $\delta>0$, such that

$$
\Lambda\left(\mathscr{N}_{\delta}(t)\right) \subset \mathfrak{N}_{\varepsilon}(\Lambda(t))
$$

(iii) $\Lambda$ is said to be pseudo-continuous at $t \in T$, if

$$
\bigcap_{\varepsilon>0} \overline{\Lambda\left(\Re_{\varepsilon}(t)\right)}=\Lambda(t) \text {. }
$$

(iv) If $T$ is a domain in $\boldsymbol{R}^{n}$, then, $\Lambda$ is said to be measurable, if for any closed subset $F \subset Z$, the set

$$
\Lambda^{-1} \equiv\{t \in T \mid \Lambda(t) \cap F \neq \varnothing\}
$$

is (Lebesgue) measurable.

If in (i)-(iii), the mentioned properties hold for all $t \in T$, we simply say $\Lambda$ is continuous, upper semi-continuous and pseudo-continuous, respectively.

Proposition A.2. Let $Z$ be a complete metric space and $\Lambda: T \rightarrow Z$ take closed subset values. Then,

(i) If $T$ is a metric space, then, the following implication chain holds

$\Lambda$ is continuous $\Longrightarrow \Lambda$ is upper semicontinuous

$$
\begin{aligned}
& \Longleftrightarrow \forall \text { closed set } F \subset Z, \Lambda^{-1} \text { is closed in } T \\
& \Longrightarrow \Lambda \text { is pseudo-continuous } \\
& \Longleftrightarrow G(\Lambda) \equiv \text { the graph of } \Lambda \text { is closed in } T \times X .
\end{aligned}
$$

(ii) If $T$ is a domain in $\boldsymbol{R}^{n}$, then,

$\Lambda$ is pseudo-continuous $\Longrightarrow \Lambda$ is measurable. 
THEOREM A.3. ([12]) Let $T$ be a locally compact metric space, $U$ be a Polish space, $X$ be a complete metric space, $\Lambda: T \rightarrow 2^{U}$ be measurable taking closed subset values, $f: T \times U \rightarrow X$ be measurable in $t$, locally uniformly continuous in $x$ and $\bar{f}: T \rightarrow X$ be measurable with

$$
\bar{f}(t) \in f(t, \Lambda(t)), \quad \text { a.e. } t \in T .
$$

Then, there exists a measurable function $u: T \rightarrow U$, such that

$$
\left\{\begin{array}{l}
u(t) \in \Lambda(t), \\
\bar{f}(t)=f(t, u(t)), \quad \text { a.e. } t \in T .
\end{array}\right.
$$

THEOREM A.4. Let $T$ be a domain in $\boldsymbol{R}^{n}, X$ be a metric space, $U$ be a Polish space, $\Gamma: T \times X \rightarrow 2^{U}$ be pseudo-continuous and $\bar{x}: T \rightarrow X$ be measurable. Then, $\Gamma(\cdot, \bar{x}(\cdot)): T \rightarrow 2^{U}$ is measurable.

The proof follows easily from the definition.

\section{REFERENCES}

[1] N.U. Ahmed And K. L. Teo, Optimal Control of Distributed Parameter Systems, North Holland, New York, 1981.

[2] E. Asplund, Averaged norms, Israel J. Math., 5 (1967), 227-233.

[3] A.V. Balakrishnan, Applied Functional Analysis, Springer-Verlag, New York, 1976.

[4] L.D. Berkovitz, Optimal Control Theory, Springer-Verlag, New York, 1974.

[5] N. Bourbaki, General Topology, Addison-Wesley Reading, 1966.

[6] F.H. Clarke, Optimization and Nonsmooth Analysis, Wiley, New York, 1983.

[7] R.F. Curtain AND A. J. Pritchard, Infinite Dimensional Linear Systems Theory, Lecture Notes in Control and Information Sciences, Vol. 8, Springer-Verlag, New York, 1981.

[8] M.M. DAY, Strict convexity and smoothness of normed spaces, Trans. Amer. Math. Soc., 78 (1955), 516-528.

[9] I. Ekeland, Nonconvex minimization problems, Bull Amer. Math. Soc. (New Series), 1 (1979), 443-474.

[10] H.O. FAtToRini, The time optimal control problem in Banach spaces, Appl. Math. Optim., 1 (1974), 163-188.

[11] H.O. FATTORINi, A unified theory of necessary conditions for nonlinear nonconvex control systems, Appl. Math. \& Optim., 15 (1987), 141-185.

[12] C. J. Himmelberg, M.Q. Jacobs and F.S. Van Vleck, Measurable multifunctions, selectors and Filippov's implicit functions lemma, J. Math. Anal. Appl., 25 (1969), 276-284.

[13] X. J. Li AND Y. YAO, Time optimal control of distributed parameter systems, Sicentia Sinica, 24 (1981), 455-465.

[14] X. J. LI AND Y. YAO, Maximum principle of distributed parameter systems with time lags, Distributed Parameter Systems, Lecture Notes in Control and Information Sciences, Springer-Verlag, New York Vol.. 75, 410-427, 1985. 
[15] X. J. Li AND J. Yong, Necessary conditions of optimal control for distributed parameter systems, SIAM J. Control \& Optim., to appear.

[16] A. PAZY, Semigroups of Linear Operators and Applications to Partial Differential Equations, Springer-Verlag, New York, 1983.

[17] L.S. Pontryagin, V. G. Boltyanskit, R. V. Gamkrelidze and E. F. Mischenko, Mathematical Theory of Optimal Processes, Wiley, New York, 1962.

[18] J. WARGA, Optimal Control of Differential and Functional Equations, Academic Press, New York, 1972.

[19] Y. YAO, Maximum principle for semilinear distributed systems (II) -time optimal control problems, Chin. Math. Ann., 4A (1983), 781-792, (in Chinese).

[20] E. Zeidler, Nonlinear Functional Analysis and Its Applications, I, SpringerVerlag, New York, 1986.

Department of Mathematics

FUDAN UNIVERSITY

SHANGHAI 200433

CHINA 